\title{
Preparation of biodiesel fuel from Urtica dioica seeds oil and study different Properties
}

\author{
Osamah M.M. Taha \\ Deparetment of Chemistry \\ College of Education For Pure \\ Science \\ University of Mosul \\ osamahmohamed81@gmail.com \\ DOI: 10.33899/edusj.1999.163324
}

\author{
Khalid S. Tawfiq \\ Deparetment of Chemistry \\ College of Education For Pure \\ Science \\ University of Mosul \\ Khalidtawfiq@gmail.com
}

\author{
Subhi M. Jarullah \\ Deparetment of Chemistry \\ College of Education For Pure \\ Science \\ University of Mosul \\ subhijarullah@gmail.com
}

Received

04/ 09 / 2018

\author{
Accepted \\ 17 / 11 / 2018
}

\begin{abstract}
Recently,biofuels are considered as an important alternative energy resource and could be a substitute for fossile fuels, due to the fact the increasing awarenees of the environmental pollution issues and depletion of fossil fuel resources (crude oil, natural gas and coal). The greatest motivation for producing biofuels is the clobal warming caused by the continuous burning of fossile fuels.

In this study,fatty acid methyl esters,known as biodiesel fuel derived from triglycerides(virgin vegetable oil of nettle), by transesterifacation with methanol,show the promising yield and better quality of biodiesel. This paper presents the development for the production of biodiesel from the vegetable oil having the high yield $91 \%$ where the transesterification reaction is promoted by finding an optimal conditions.

Clearly the process of transesterification is directly affected by the mode of reaction condition including: molar ratio of alcohol to oil, type of alcohol, amount of $\mathrm{KOH}$, reaction time and temperature. In the present paper,various conditions of preparation of biodiesel have been described to obtain the best quality and high yield.
\end{abstract}

Key words: biodiesel,Urtica dioica, Transesterification 


\title{
تحضير وقود الايزل الحيوي من زبت بذور نبات القربص (Urtica dioica) ودراسة صفاته المختلفة
}

صبحي محسن جارالله

$$
\begin{aligned}
& \text { قسم الكيمياء } \\
& \text { كلية التربية للعلوم الصرفة } \\
& \text { جامعة الموصل }
\end{aligned}
$$

subhijarullah@gmail.com

DOI: 10.33899/edusj.1999.163324
خالد سعيد توفيق

$$
\text { قسم الكيمياء }
$$

كلية التربية للعلوم الصرفة

جامعة الموصل

Khalidtawfiq@gmail.com
أسامة محمد مجيد طه

$$
\text { كلية التربية للعلوم الصرفة الكيمياء }
$$

osamahmohamed81@gmail.com

\author{
القبول

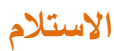 \\ 2018 / $11 / 17$ \\ 2018 / 09 / 04
}

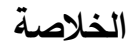

في الاونة الاخيرة يعد الوقود الحيوي مصدرا هاما للطاقة البديلة ويمكن ان يكون بديلا عن الوقود الاحفوري، وذلك بسبب

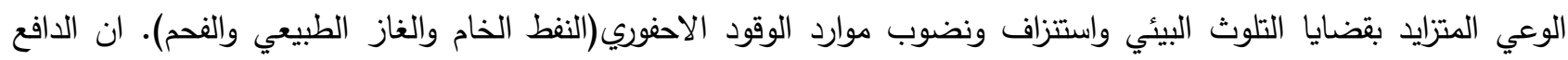
الاكبر لانتاج الوقود الحيوي هو الاحتباس الحراري العالمي الناجم عن الاحتراق المستمر للوقود الاحفوري.

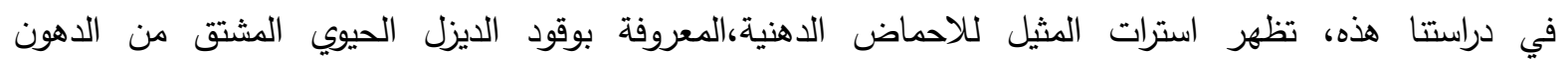
الثلاثية،ذورنبات القريص عن طريق تفاعل انتقال الاسترة بوجود الميثانول، انتاج واعد لنوعية افضل من وقود الديزل الحيوي. تم

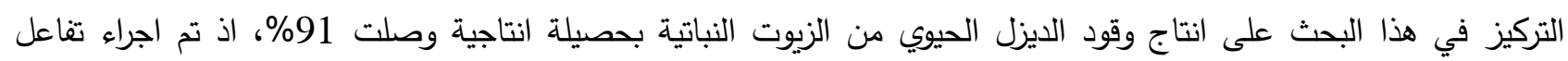

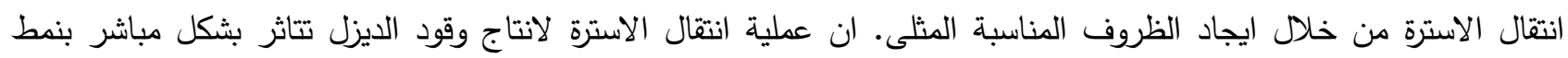
ظروف التفاعل والتي تثمل: النسبة المولارية من الكحول الى الزيت، نوعية الكحول المستعمل،كمية القاعدة المضافة،زئن التفاعل ودرجة الحرارة. اذ تم تحديد هذه المتغيرات من خلال هذه الدراسة.

الكلمات المفتاحية الدالة: (ديزل حيوي، نبات القريص (Ud)، تفاعل انتقال الاسترة) 


\section{المقدمة}

الوقود الحيوي هو نوع من أنواع وقود الكتلة الحيوية التي زاد الأهتمام بها خلال العقدين الأخيرين ويشتق أو يمكن الحصول علية من المواد العضوية الحيوية أو مخلفاتها(1) ويشير الى الوقود السائل او الغازي المستعمل في الغالب في وسائل النقل المتعددة والذي ينتج معظمه من مصادر

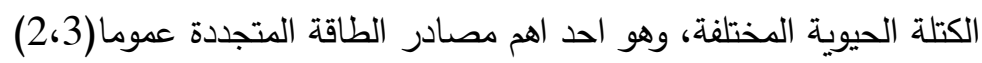
تم تثخيص أكثر من350 نوعاً من المحاصيل التي تحتوي على زيوت من بينها زهرة الثمس ، والقرطم ، وفول الصويا

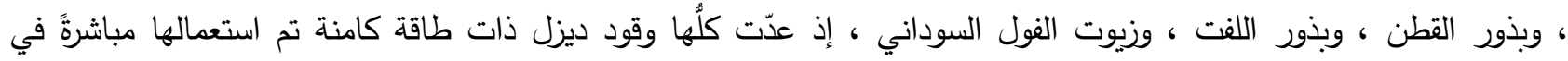
المحركات بالنسبه لمحرك الديزل (1).

اوضحت الدراسات ان الوقود الحيوي سوف يكون ذا استعمال واسع في المستقبل وذلك لما له من مزايا وفوائد كثيرة يمكن تلخيصها بما ياتي: (2، (1، (2) 1-يمكن الحصول عليه بسهولة من المصادر الثائعة للكتلة الحيوية المتجددة اذ انه متاح محليا

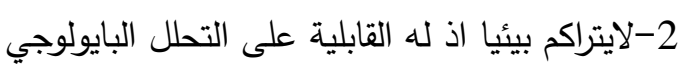
3- وقود مستدام لاينضب 4- يمكن ان يحل محل المحروقات البترولية في توفير الطاقة. 5-تيتم تدوير غاز ثنائي اوكسيد الكاربون الذي ينتج اثناء عملية الاحتراق من خلال الطبيعة. والديزل الحيوي هو أحادي الكيل الاستر للحوامض الدهنية المشتق من زيوت النباتات وشحوم الحيوانات، ويتم إنتاجه

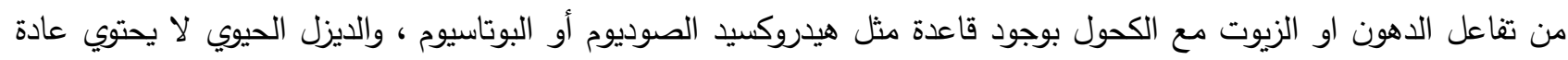

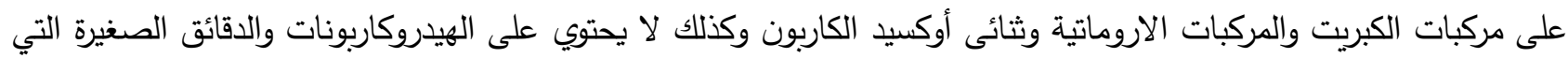
تلوث الجو وتسبب مرض السرطان(4). تنسر مراجع الادبيات اختلاف الطرائق في تحضير وقود الديزل الحيوي من مواد اولية مختلفة وطرائق وحفازات مختلفة،

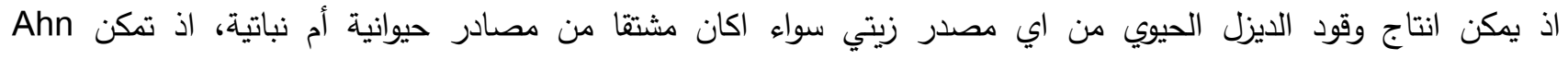

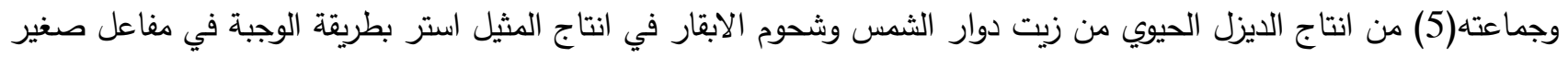
وباستعمال قاعدة من هيدروكسيد الصوديوم والبوتاسيوم. كما ناقش Gerpen (6) تأثير الزمن ودرجة حرارة التفاعل على حصيلة الديزل الحيوي المنتج من نبات الجاتروفا باستعمال القاعدة. حضر Magin (7) الديزل الحيوي من فضلات زيت الطبخ باستعمال الكحولات المختلفة مثل الميثانول والايثانول وبوجود قاعدة ، ووجد أن الكحول المستعمل يؤثر على خواص الوقود الناتج. 


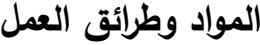

تم استعمال مواد كيميائية مختلفة في الدراسة الحالية وكما موضح في الجدول ادناه:

الجدول (2-1) المواد الكيميائية المستعملة

\begin{tabular}{|c|c|c|}
\hline المجهز & & المواد الكيميائية \\
\hline $\mathrm{BDH}$ & Methanol & 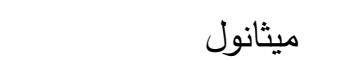 \\
\hline BHD \& Tedia & Absolute Ethanol & ايثانول مطلق \\
\hline Fluka & n- Hexane & هكسان طبيعي \\
\hline $\mathrm{BDH}$ & Isopropyl alcohol & كحول ايزوبروبيلي \\
\hline Merck & Hydchloric acid & حامض الهيدروكلوريك \\
\hline Tedia & Acetone & اسيتون \\
\hline Merck & Potassium hydroxide & هيدروكسيد البوتاسيوم \\
\hline $\mathrm{BDH}$ & lodine & يود \\
\hline FLUKA & Zinc chloride & كلوريد الخارصين \\
\hline $\mathrm{BDH}$ & Phenophthalene & دليل فينولفثالين \\
\hline $\mathrm{BDH}$ & Potassium iodide & يوديد البوتاسيوم \\
\hline $\mathrm{BDH}$ & $\begin{array}{l}\text { Sodium thio } \\
\text { sulphate }\end{array}$ & ثايوسلفات الصوديوم \\
\hline
\end{tabular}

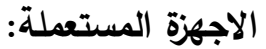

1- مقياس اللزوجة ( Ostwald Viscometer) (جامعة الموصل).

2- مقياس معامل الانكسار (كلية التربية للعلوم الصرفة/جامعة الموصل).

3- مقياس نقطة الوميض (جامعة الموصل).

4- مقياس العدد السيتاني (جامعة الموصل).

5- جهاز كروموتوغرافيا الغاز GC (وزارة العلوم والتكنولوجيا/بغداد).

اختيار انموذج النبات قيد الدراسة:

استعمل في تحضير وقود الديزل الحيوي في دراستتا هذه زيت بذور نبات القربص اسمه الاجنبي Nettle

Urtica dioica ، وذلك لاحتوائه على كميات مناسبة من الزيوت، وكذلك لانتشار هذا النبات في محافظة نينوى بكثافة عالية. 
تم جني هذا النبات من الاراضي الخصبة وجوانب الطرق في مناطق مختلفة من محافظة نينوى، وقد تم عزل البذور وتتظيفها من الشوائب وتهيئتها لعملية استخلاص الزيوت منها، والتي استعملت كمادة اولية لانتاج وقود الديزل الحيوي.

\section{Extractions of vegetable}

\section{استخلاص الزيوت النباتية}

تم استخلاص الزيوت المستعملة قيد الدراسة من انموذج بذور نبات القريص (Ud) باستعمال جهاز الاستخلاص

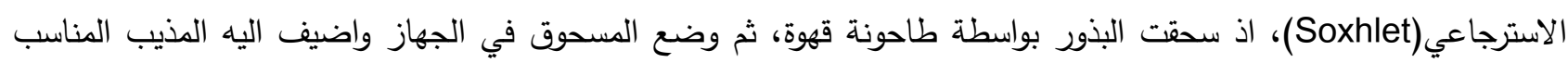
بنسبة (1-3 )(بذور: مذيب) اذ استعمل مذيب الهكسان وذلك لملائمته للاستخلاص بعد الدراسة ومقارنته مع غيره من المذيبات المتوفرة مثل مذيب البتروليوم ايثر ذو مدى الغليان (60-80)خ والذي كانت نسبة استخلاصه للزيت اقل من الهكسان.

بعدها تم اجراء التصعيد الحراري لمدة 24 ساعة باستعمال حمام مائي وبدرجة غليان المذيب، ثم ترشيح الناتج، واسترجع المذيب

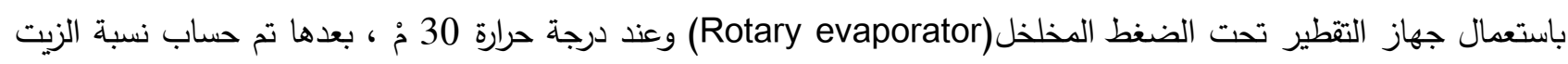
المستخلص وتم الحصول على نسبة (31.0) \% ؛ بعدها حددت بعض الخواص المهمة من الزبت لمعرفة مدى ملائمتها للاستعمال

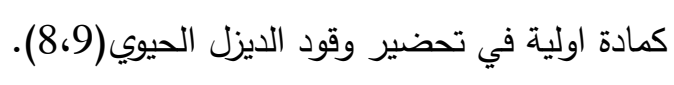

\section{Transesterification reaction}

تفاعل انتقال الاسترة

تم مفاعلة انموذج الزيت المستعمل في دراستا هذه مع كحول الميثانول خلال تفاعل انتقال الاسترة وبوجود القاعدة وكما

وضعت كمية مناسبة من الزيت المستعمل في هذه الدراسة (Ud) في دورق ثلاثي العنق سعة 250 مل متصل بمكثف وترك

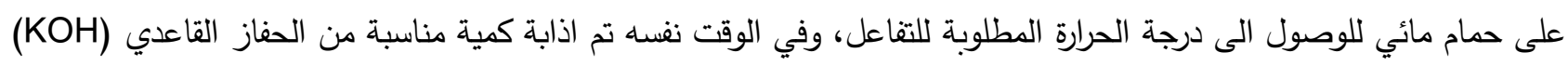
في كحول الميثانول ثم اضيف المزيج الى دورق التفاعل. بعدها اجري التصعيد الحراري عند درجات حرارية مختلفة لاختيار الدرجة الحرارية الامثل ولفترات زمنية متباينة مع التحريك

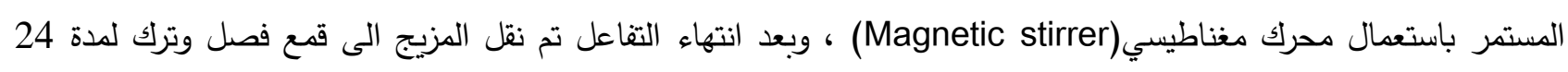
ساعة، لوحظ تكون طبقتين. الطبقة العليا تمثل طبقة المثيل استر(Methyl ester) والطبقة السفلى تمثل الكليسيرول

.(Glycerol)

تم فصل طبقة المثيل استر والتي تمثل وقود الديزل الحيوي(BD) ووضعت في جهاز التقطير تحت الضغط المخلخل( Rotary (evaporator من القاعدة.

في النهاية ينقى مثيل الاستر ويجف باستعمال كبريتات المغنيسيوم او كلوريد الكالسيوم وبعد ذلك يرشح ويحفظ لاجراء الفحوصات عليه(10) بعدها تم حساب الحصيلة الانتاجية وفق العلاقة الاتية:

Production proceeds $=$

Wt.of BD

Wt. of Oil

*100 


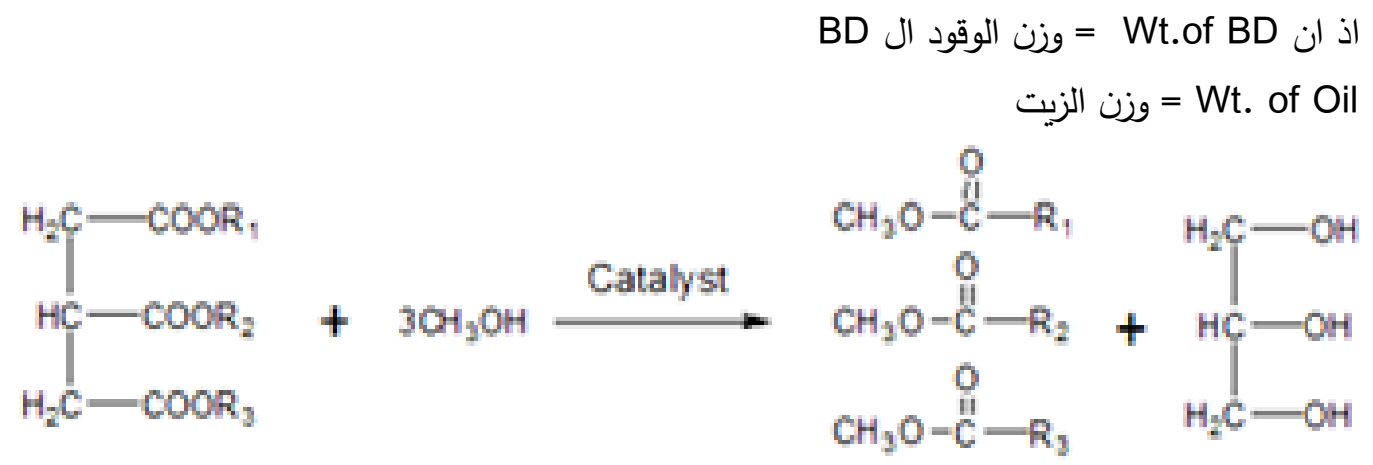

Triglyceride Methanol Methyl Esters Glycerin

يمكن توضيح المعادلة العامة لتفاعل انتقال الاسترة كما يلي:

تم اجراء تفاعل انتقال الاسترة(TE) على زيت القريص بوجود قاعدة اذ تم التفاعل باستعمال ظروف التفاعل الاتية: تم استعمال نسب وزنية من القاعدة KOH تراوحت بين ( 0.75 و 1.5)\% وبزيادة بلغت زيت)، وتم ايضا تغيير نسب الميثانول المستعملة في التقاعل من (3:1) الى (12:1) وبزيادة مولية بلغت(3) مواتئ (3) مول لكل

قراءة(زيت:ميثانول).

وتم اجراء التفاعل بازمان مختلفة تراوحت بين 30 الى 120 دقيقة وبزيادة 30 دقيقة لكل قراءة، كذلك تم استعمال

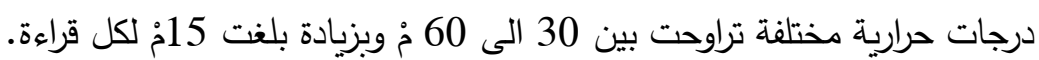

تحديد الخواص الفيزيائية والكيميائية لوقود الديزل الحيوي

Determining Physiochemical Properties for Biodiesel

تم قياس معظم الخصائص الفيزيائية والكيميائية لزيت Ud وكذلك وقود الديزل الحيوي المحضر منه طبقا للمواصفات القياسية الامريكية)

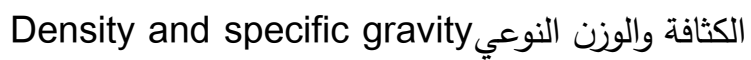

Kinematic Viscosity (Kv) اللزوجة الكيناميتية

درجة الضبابية (CP) pour point(pp) درجة الانسكاب

درجة الوميض (FP)

Refractive Index معلم الانكسار

Acid Value (AV) قيمة الحامضية رقم اليود lodine Number (IN)

Saponification Value (SV) قيمة الصوبنة

Cetane Index (Cl) معامل السيتان

Cetane Number(CN) عدد السيتان GC التحليل باستعمال كروموتوغرافيا الغاز 


\section{النتائج والمناقشة}

تم دراسة الظروف التجريبية المؤثرة على التفاعل مثل نسبة تركيز القاعدة المستعملة وكذلك النسبة المولارية للميثانول فضلا عن زمن التفاعل، كذلك تم اجراء تفاعل انتقال الاسترة على الزيت بوجود الهكسان كمذيب مشترك ودراسة العوامل المؤثرة على التفاعل.

تم بعد ذلك تحديد الخواص الفيزيائية والكيميائية للوقود الناتج ومقارنتها مع مواصفات وقود(BD) المحضر عالميا فضلا عن المواصفات القياسية (ASTM). من خلال ملاحظة المواصفات الفيزيائية والكيميائية لزيت القريص نلاحظ ان كثافة الزيت (Udo) مقاربة لبعض الزيوت

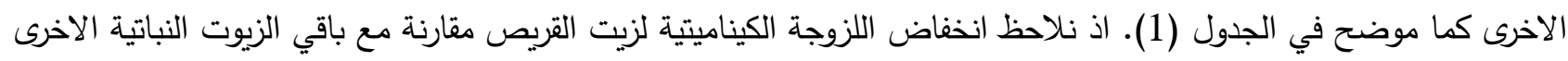

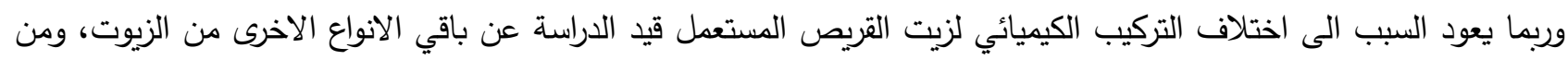

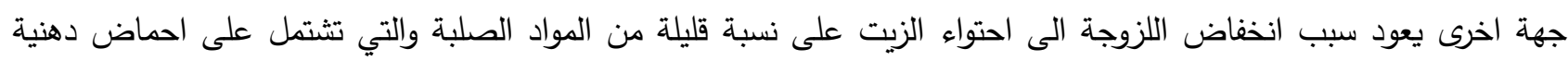
مشبعة عالية الوزن الجزيئي. اما فيما يتعلق بقيمة الحامضية(AV) والتي تعد من اهم الصفات الكيميائية للزيت لانها تحدد الطريقة الملائمة لتحويل الزيت الى وقود الديزل الحيوي فقد بلغت (12.91 ملغم KOH/غم زيت) وهذه القيمة اعلى بكثير من تلك الملاحظة لباقي الزيوت النباتية

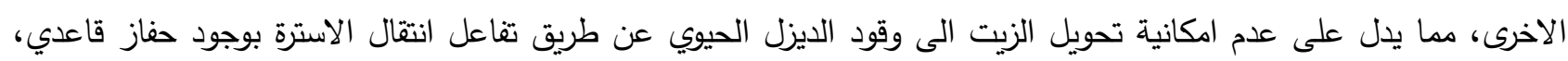

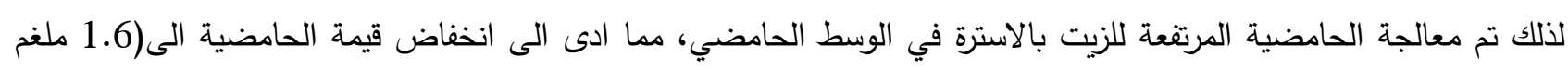
/KOH

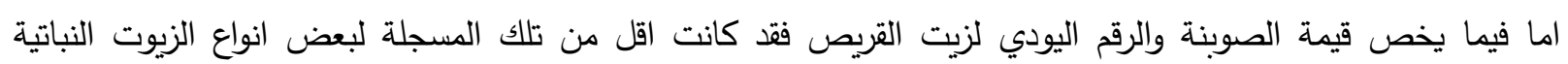

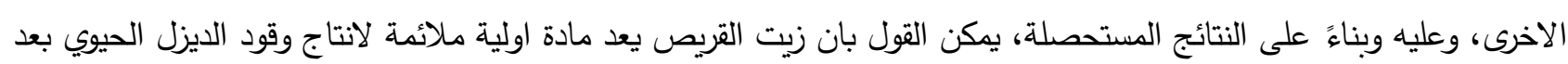
اجراء التحويرات الملائمة عليه. 
جدول(1) : المواصفات الفيزيائية والكيميائية لزيت القريص بالمقارنة مع مواصفات الزيوت الاخرى(13،11،11، أندات

\begin{tabular}{|c|c|c|c|c|c|c|c|c|c|}
\hline زيت & زيت & زليت & فوليت & زلخيت & زبت & زيت & زهرة زيت & القريص زيت & الفيزيائية الكفات \\
\hline 0.914 & 0.920 & 0.911 & 0.913 & 0.960 & 0.912 & 0.923 & 0.920 & 0.9010 & الكثافة(غم/سم 3 \\
\hline 4 & 0 & 5 & 8 & 0 & 0 & 6 & 0 & & 15.6) \\
\hline 31.30 & 35.40 & 37.00 & 32.90 & $\begin{array}{c}227.0 \\
0\end{array}$ & 36.00 & 27.20 & 32.60 & 22.6 & (اللزوجة \\
\hline- & 11.00 & 2.92 & 0.20 & 0.63 & 0.07 & - & 2.92 & 12.91 & قيمة الحامضية \\
\hline 190.2 & 209.0 & 197.0 & 202.0 & 202.7 & 207.7 & 188.7 & 201.0 & 182.52 & $\begin{array}{c}\text { قيمة الصوبنة } \\
\text { mg KOH/g } \\
\text { Oil }\end{array}$ \\
\hline 139.8 & $\begin{array}{c}110 . \\
0\end{array}$ & 108.0 & 101.0 & 88.7 & 113.2 & 156.7 & 140.0 & 111.2 & اليود(100ملفغم غلغ زيت \\
\hline- & - & - & - & - & - & - & - & $\begin{array}{c}1.4677 \\
0\end{array}$ & معامل الانكسار \\
\hline 260 & 240 & 246 & 254 & 230 & 250 & 241 & 274 & 186 & درجة الوميض مُ \\
\hline $6.7-$ & $3.0^{-}$ & $31.7-$ & $12.2^{-}$ & - & $14.0^{-}$ & $15.0^{-}$ & $15.9-$ & $17-$ & درجة الانسكاب \\
\hline 18.3 & 2.0 & $3.9-$ & $3.9-$ & $3.0^{-}$ & 2.8 & 1.7 & 7.2 & $3-$ & درجة الضبابية \\
\hline
\end{tabular}

Influence The Concentration ratio of Potasium Hydroxide تأثير نسبة تركيز هيدركسيد البوتاسيوم تمت دراسة تاثير نسبة تركيز القاعدة على مواصفات الديزل الحيوي المضركيز هيدروكانت النتائج كما في الجدول(2) 
الجدول(2): تاثيرنسبة تركيز هيدروكسيد البوتاسيوم على المواصفات الفيزيائية والكيميائية للديزل الحيوي المحضر من زيت

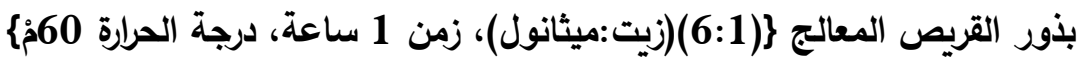

\begin{tabular}{|c|c|c|c|c|}
\hline 1.50 & 1.25 & 1.0 & 0.75 & نسبة الحفاز المواصفات \\
\hline 79 & 80 & 91.0 & 75.5 & حصيلة الانتاج (\%) \\
\hline 0.88994 & 0.84998 & 0.83684 & 0.88970 & الكثافة(غم/سم33،15:6م \\
\hline 7.2356 & 7.0102 & 4.6943 & 7.2360 & اللزوجة (ملم²ثا)،40م \\
\hline 0.89073 & 0.850743 & 0.837592 & 0.890499 & الوزن النوعي،15.6م \\
\hline 1.7 & 1.2 & 0.73 & 1.64 & قيمة الحامضية(ملفمKOH/غم) \\
\hline 165 & 139.5 & 122 & 162 & لدرجة الوميض مْ \\
\hline $12-$ & $11-$ & $5-$ & $13-$ & درجة الانسكاب مُ \\
\hline 5 & 4 & 1 & 4 & درجة الضبابية مُ \\
\hline
\end{tabular}

يعود سبب انخفاض حصيلة ال (BD) المنتج عند استعمال التركيز الاقل من(1.0)\% الى عدم كفاية تلك التراكيز لاكمال تحويل الزيت المستعمل الى الاستر المقابل، كما قد تسبب التراكيز الاعلى من التركيز المذكور من الحفاز القاعدي في احتمالية تكوين الصوابين مما يؤدي ايضا الى نقصان في حصيلة الاتتاج.(14) وبذلك يعد تركيز (1.0)\% من الحفاز (KOH) هو الإلى التركيز التركيز الامثل لانتاج الديزل الحيوي من زيت بذور القريص المعالج عند ثبوت متغيرات التفاعل الاخرى من زمن ودرجة الحرارة والنسبة

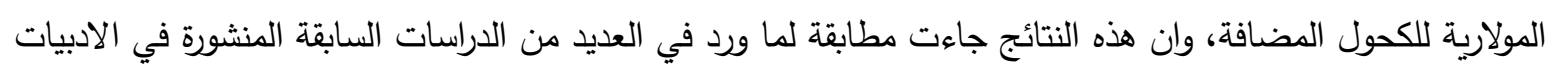
$(15 ، 16 ، 17)$

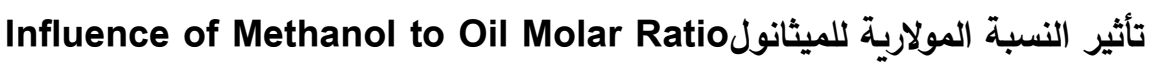
تم دراسة النسبة المولارية للميثانول على مواصفات الديزل الحيوي المحضر وكانت النتائج حسب الجدول3.

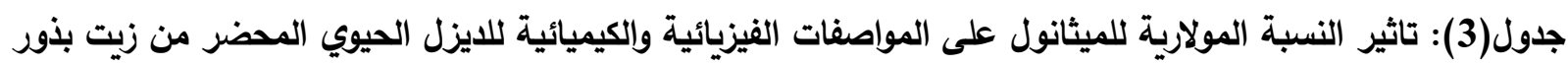

\section{القريص}

\}نسبةتركيز القاعدة(1.0)\% وزنا، زمن 1 ساعة، درجة الحرارة (60)مُ

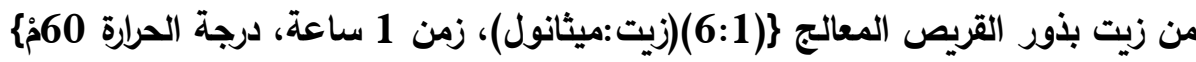

\begin{tabular}{|c|c|c|c|c|}
\hline $12: 1$ & 9:1 & $6: 1$ & $3: 1$ & النسبة المولاربية للميثانول \\
\hline 87.5 & 90.5 & 91.0 & 78 & حصيلة الانتاج (\%) \\
\hline 0.84450 & 0.89220 & 0.83684 & 0.89920 & الكثافة(غم/سم33،15:6م \\
\hline 4.71200 & 4.67003 & 4.6943 & 7.2032 & اللزوجة(ملم²ثا)،40م \\
\hline 0.845258 & 0.893003 & 0.837592 & 0.900007 & الوزن النوعي،15.6مْ \\
\hline 0.98 & 0.88 & 0.73 & 2.0 & قيمة الحامضية(ملغمKOH/غم) \\
\hline 129.5 & 128 & 122 & 181.5 & درجة الوميض مُ \\
\hline 7- & $5-$ & $5-$ & $10-$ & درجة الانسكاب مْ \\
\hline 1 & 1 & 1 & $1-$ & درجة الضبابية مُ \\
\hline
\end{tabular}


يلاحظ من النتائج في الجدول اعلاه، ان تفاعل انتقال الاسترة لم يكتمل في حالة استعمال النسبة المولارية الاقل من (6:1)، وان حصيلة انتاج الديزل الحيوي ازدادت بزيادة النسبة المولارية للميثانول من (3) الى (6) لتعطي المثيل استر الناتج

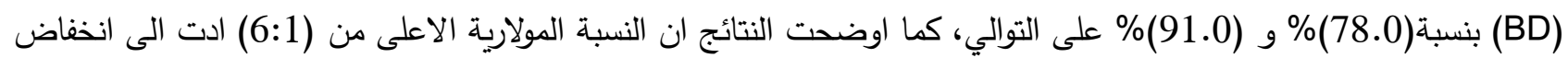
حصيلة ال (BD) المنتج، فعند ارتفاع النسبة الى (12:1) فان كمية الميثانول العالية سوف تؤثر على عملية فصل الكليسيرين بسبب زيادة ذوبان الاخير في الميثانول مما يزيد في صعوبة عملية الفصل، كما ان وجود الكليسرين في محلول التفاعل يزيح

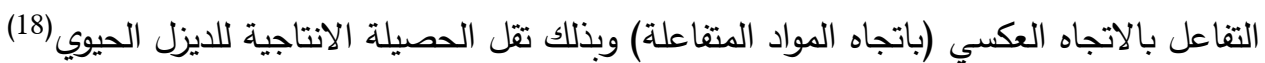
وعليه تعد نسبة(6:1) هي النسبة الامثل لانتاج وقود ال (BD) من زيت بذور القريص المعالج وباعلى حصيلة، وهذا ماتم اثباته

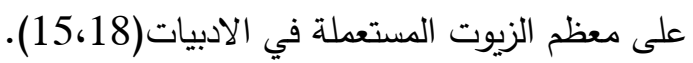
Influence of Reaction Time تاثير زمن التفاعل معظي التبون تمت دراسة تاثير زمن التفاعل على مواصفات الديزل الحيوي المحضر كما مبين في الجدول 4. جدول(4): تاثير زمن التفاعل على المواصفات الفيزيائية والكيميائية للايزل الحيوي المحضر من زيت بذور القريص

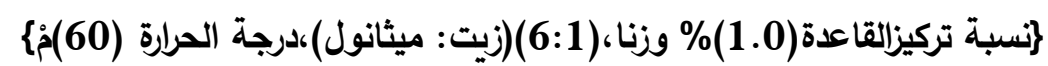

\begin{tabular}{|c|c|c|c|c|}
\hline 120 & 90 & 60 & 30 & المواصفات \\
\hline 87.5 & 90.0 & 91.0 & 81 & حصيلة الانتاج (\%) \\
\hline 0.80022 & 0.80122 & 0.83684 & 0.82760 & الكثافة(غم/سم33)،15:6م \\
\hline 4.25300 & 4.61100 & 4.6943 & 7.4200 & اللزوجة (ملم²/ا)،40م \\
\hline 0.800938 & 0.80193 & 0.837592 & 0.82834 & الوزن النوعي،15.6م \\
\hline 0.70 & 0.68 & 0.73 & 0.79 & قيمة الحامضية(ملفمKOH/غم) \\
\hline 130 & 130 & 122 & 134 & درجة الوميض مْ \\
\hline 7- & $5-$ & $5-$ & 9- & درجة الانسكاب مُ \\
\hline $1-$ & $1-$ & 1 & $2-$ & درجة الضبابية مُ \\
\hline
\end{tabular}

نلاحظ من الجدول(4)، ان زيادة زمن التفاعل من (30) دقيقة الى (60) دقيقة ادى الى زيادة في الحصيلة الانتاجية للا (60) الناتج عند ثبوت المتغيرات الاخرى من نسبة الحفاز والنسبة المولارية للكحول المستعمل ودرجة الحرارة، وان الزمن (BD)

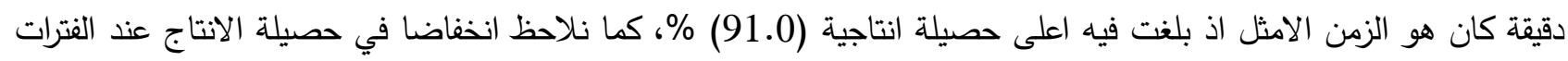
الزمنية الاعلى من (60) دقيقة ويعزى سبب ذلك الى احتمالية تحلل الديزل الحيوي الناتج مع زيادة زمن التفاعل المستعمل وازاحة

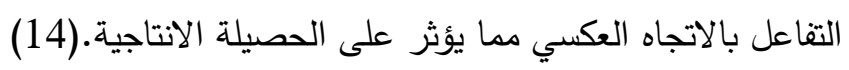
على ضوء ذلك تم تثبيت زمن التفاعل ساعة واحدة كافضل زمن لاجراء تفاعل انتقال الاسترة على زيت بذور القريص المعالج لانتاج اعلى حصيلة من وقود الديزل الحيوي، كما طابقت هذه الدراسة ماجاء به العديد من الباحثين في الدراسات المنشورة في الادبيات(17) 


\section{Influence of Temperature}

تاثير درجة الحرارة

يمكن توضيح تاثير درجة الحرارة على مواصفات الديزل الحيوي المحضر كما في الجدول الاتي:

جدول(5): تاثير درجة الحرارة على المواصفات الفيزيائية والكيميائية للديزل الحيوي المحضر من زيت بذور القريص

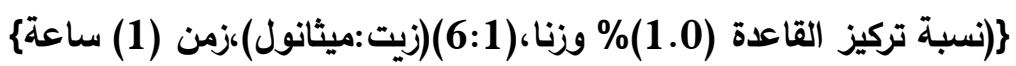

\begin{tabular}{|c|c|c|c|c|}
\hline 75 & 60 & 45 & 30 & المواصفات \\
\hline 78.5 & 91.0 & 82.5 & 79 & حصيلة الانتاج (\%) \\
\hline 0.80212 & 0.83684 & 0.81012 & 0.82210 & الكثافة(غم/سم33)،15:6 \\
\hline 4.15300 & 4.6943 & 5.0220 & 6.9400 & اللزوجة (ملم²/ثا)،40م \\
\hline 0.802840 & 0.837592 & 0.810847 & 0.822838 & الوزن النوعي،15.6مْ \\
\hline 1.10 & 0.73 & 0.77 & 0.80 & قيمة الحامضية(ملفمKOH/غم) \\
\hline 139 & 122 & 138 & 136 & درجة الوميض مُ \\
\hline $5-$ & $5-$ & 7- & 7- & درجة الانسكاب مُ \\
\hline 3- & 1 & $1-$ & $2-$ & درجة الضبابية مُ \\
\hline
\end{tabular}

نلاحظ من نتائج الجدول اعلاه ارتفاع الحصيلة الانتاجية مع زيادة درجة الحرارة الى (60)خ وقد يرجع ذلك الى زيادة سرعة تفاعل انتقال الاسترة بسبب انخفاض لزوجة الزيت وتحسين عملية الامتزاج بين المواد الاولية وبالتالي تزداد

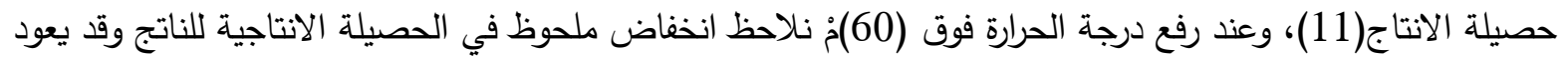
السبب الى تاثر تركيب الزيت مع ارتفاع درجة الحرارة اعلى من (60)مْ وهذا مايفسر ارتفاع قيمة الحامضية، ولذلك فان درجة حرارة (60)؛ اعتبرت هي الدرجة الحرارية المثلى للتفاعل(10)، وبذلك يمكن مقارنة مواصفات الديزل الحيوي المنتج من زيت بذور القريص مع الديزل القياسي وانواع اخرى كما مبينة في الجدول 6. 
الجدول(6) المواصفات الفيزيائية والكيميائية للايزل الحيوي المنتج من زيت بذور القريص بالمقارنة مع بعض انواع الديزل الحيوي الاخرى والديزل القياسي(20،19،18)

\begin{tabular}{|c|c|c|c|c|c|c|c|}
\hline $\begin{array}{c}\text { BD } \\
\text { قياسي }\end{array}$ & $\begin{array}{l}\text { BD } \\
\text { النخيل }\end{array}$ & $\begin{array}{c}\text { BD } \\
\text { جاتروفا }\end{array}$ & $\begin{array}{l}\text { BD } \\
\text { القرطم }\end{array}$ & $\begin{array}{c}\text { BD } \\
\text { الخروع }\end{array}$ & $\begin{array}{c}\text { BD } \\
\text { القطور }\end{array}$ & $\begin{array}{c}\text { BD } \\
\text { القريص }\end{array}$ & المواصفات الفيزيائية \\
\hline- & - & - & - & - & - & 91.0 & حصيلة الانتاج\% \\
\hline $\begin{array}{c}-0.860 \\
0.900\end{array}$ & 0.877 & 0.880 & 0.862 & 0.899 & 0.886 & 0.8368 & الكثافة(غم/سم)،15.6مُ \\
\hline $6.00-1.90$ & 4.61 & 4.75 & 6.13 & 15.20 & 4.90 & 4.6943 & اللزوجة(ملم²ثا)،40م \\
\hline 一 & - & - & - & 一 & 0.887 & 0.8375 & الوزن النوعي،15.6م \\
\hline العظى 0.80 & 0.62 & 0.40 & 0.63 & 0.90 & 0.31 & 0.73 & الحامضية(ملفم KOH/غم) \\
\hline 一 & 一 & - & - & 176.0 & 一 & 183.9 & الصوبنة(ملفم KOH/غم) \\
\hline العظمى120.0 & 54.0 & 109.5 & 141.0 & 82.0 & - & 102.0 & رقم اليود(100ملفم2ا/غم) \\
\hline 一 & 一 & 一 & 一 & 一 & - & 1.46168 & معامل الانكسار \\
\hline الصغرى 90 & 163 & 152 & 80 & 178 & 178 & 122 & درجة الوميض مُ \\
\hline حسب البلد & 13 & $\mathbf{0}$ & 9- & $15^{-}$ & 3 & $5-$ & درجة الانسكاب مُ \\
\hline حسب البلد & 14 & 5 & 9 & $13-$ & 6 & 1 & درجة الضبابية مُ \\
\hline $47.0-40.0$ & 70.0 & 55.7 & 52.0 & 一 & 57.1 & 69.0 & عدد السيتان \\
\hline الصغرى 47.0 & 一 & 一 & 一 & - & - & 73.68 & معامل السيتان \\
\hline
\end{tabular}


User Name:

Sample Name $=$ st mix FAME ( 80 PPM )

Injection Volume $=1 \mathrm{uL}$

Tem Injector $=280 \mathrm{C}$

Tem Detector (FID ) $=330 \mathrm{C}$

Column Oven ( ZB -5MS $)=100-150 \mathrm{C}(8 \mathrm{C} / \mathrm{MIN}) 150-300 \mathrm{C}(10 \mathrm{C} / \mathrm{MIN})$

Pressure $=100 \mathrm{kpa}$

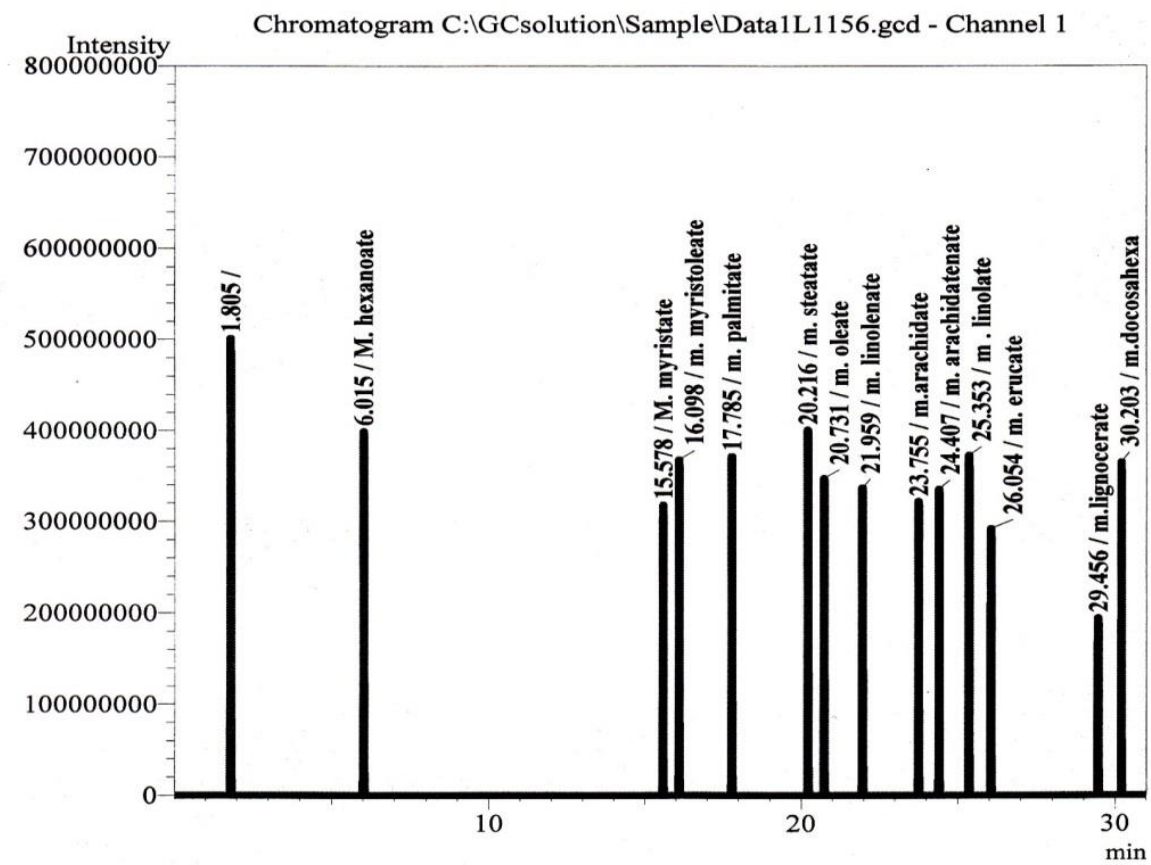

Peak Table - Channel 1

\begin{tabular}{|c|c|c|c|c|c|}
\hline \\
\hline Peak\# & Ret.Time & Area & Area\% & Height & Name \\
\hline 1 & 1.805 & 485875396 & 10.1104 & 87137892 & \\
\hline 2 & 6.015 & 396752962 & 8.2559 & 89239989 & M. hexanoate \\
\hline 3 & 15.578 & 313963825 & 6.5332 & 08865725 & M. myristate \\
\hline 4 & 16.098 & 355729926 & 7.4022 & 43478495 & m. myristoleate \\
\hline 5 & 17.785 & 367192392 & 7.6408 & 63031610 & m. palmitate \\
\hline 6 & 20.216 & 389004967 & 8.0947 & 90436786 & m. steatate \\
\hline 7 & 20.731 & 336512155 & 7.0024 & 19217179 & m. oleate \\
\hline 8 & 21.959 & 331276170 & 6.8934 & 14482742 & m. linolenate \\
\hline 9 & 23.755 & 319297295 & 6.6441 & 96814879 & m.arachidate \\
\hline 10 & 24.407 & 329730751 & 6.8612 & 27025272 & m. arachidatenate \\
\hline 11 & 25.353 & 359544562 & & 57369289 & m. linolate \\
\hline 12 & 26.054 & 281740343 & 5.8626 & 85040260 & m. erucate \\
\hline 13 & 29.456 & 186982250 & 3.8908 & 87105543 & m.lignocerate \\
\hline 14 & 30.203 & 352097753 & 7.3267 & 53105229 & m.docosahexa \\
\hline Total & & 4805700747 & 100.000 & 22350890 & \\
\hline
\end{tabular}

الشكل 1 يوضح الكروماتوغرام الخاص بالاحماض الدهنية القياسية 

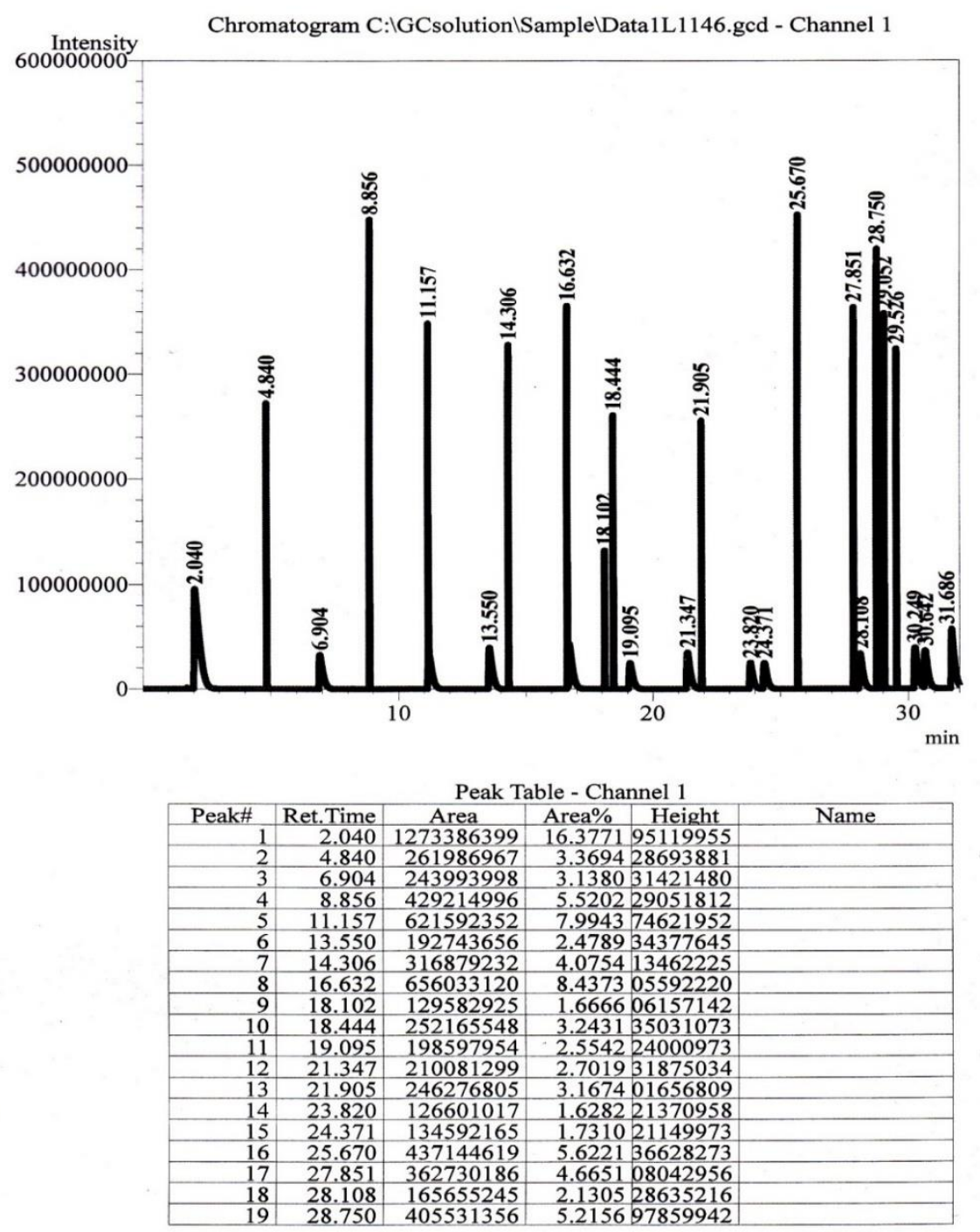

الثكل 2 يوضح الكروماتوغرام الخاص بوقود الديزل الحيوي المحضر

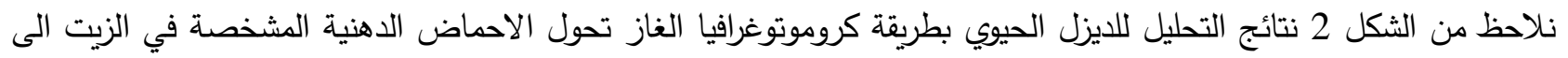

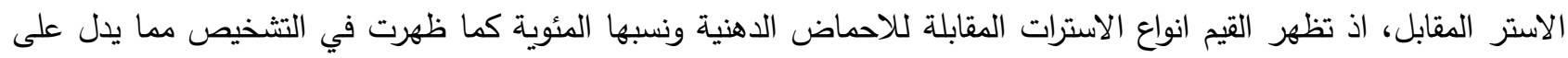
تحول الزيت بأحماضه الدهنية الى الديزل الحيوي الدقابل ونجاح عملية انتقال الأسترة.

\section{Conclusions الاستنتاجات}

1- يمكن ادراج الزيت المستخلص من بذور نبات القريص كمادة أولية مهمة في انتاج وقود الديزل الحيوي، بوصف هذه البذور مادة متجددة ومتوفرة ورخيصة وذات محتوى زيتي جيد، فضلا عن كونها غير صالحة للأستهلاك البشري.

2- الديزل الحيوي المنتج من زيت بذور القريص كان ذو مواصفات مطابقة للمواصفات المثبتة عالميا . 3- ان أفضل الظروف التجربية التي تم أستعمالها في بحثنا هذا تمثلت بما يأتي:

\} نسبة تركيز القاعدة (1.0)\% وزنا من هيدروكسيد البوتاسيوم،(6:1)(زيت:ميثانول)، درجة الحرارة(60)خهزمن(1) ساعة

Acknowledgements: University of Mosul/Edication College of pure science 


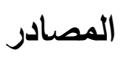

1-Pryor R. W., Hanna M. A., Schinstock J. L., Bash L. L. "Trans ASAE" 44)333-8. (1982).

2- Demirbas, A.; "Biodiesel". A realistic fuel Alternative for Diesel Engines, Springer-Verlag London Limited, PP.1-208. (2008).

3- Bart, J.C.J.; Palmeri, N.; and Cavallaro, S.; "Biodiesel Science and Technology From Soil to Oil". Woodhead Publishing Limited, PP. 1-858. (2010).

4-Aninidita k., Souti M., Elsevier ,101, 7201-7210 , (2010) .

5- Ahn E., Koncar M., Mittelbach M. and Man R., Separation Science and Technology ; 30:2021-33,(1995) •

6-Van G. Jon. Fuel Processing Technology ; 86: 1097-107, ( 2005).

7- Magin L.,.Herreros, J.M., Lisbethel I., Reyes G.C, Yolanda B., Fuel ; 87, pp.3161-3169, (2008) •

8-Fang, Z.; (2012). Intech, P. 86., 114-121. London , (2009) .

9-Achachlonei, B.F.; and Damirchi, S.A., J. Am. Oil Chem. Soc., Vol. 86, PP. 643-649. (2009).

10-Shailendra, S.; Avinash K.A.; and Sanjeev, G., Energy conversion and management, Vol. 49, No. 5, PP. 1248-1257. (2008).

11-Bankovic-llic, I.B.; Stamenkovic, O.S.; and Veljkovic, V.B., Renewable and sustainable energy reviews, Vol.16, PP. 3621-3647. (2012).

12-Singh, S.P., and Singh, D., Renewable and Sustainable Energy Reviews, Vol. 14, PP. 200216. (2010).

13-Pena, R.; et al., Ind. Eng. Chem. Res., Vol.48, PP. 1186- 1189. (2009).

14-Dennis, Y.C.; and Xuanwu, M.K.H., Applies energy, Vol. 87, PP. 1083-1095. (2010).

15-Karnwal, A.; and et al., Iranica Journal of Energy and Environment, Vol.1, No.4, PP. 352358. (2010).

16-Dorado, M.P.; Ballesteros, E., Lopez, F.J., and Mittelbatch, M., Energy and fuels, Vol. 18, PP. 77-83. (2004).

17-Abd alaziz N.A.,"PHD.Sc. Thesis, Edication College of pure science, University of Mosul(2017). \{In Arabic $\}$

18-Keera, ST.; Elsabagh, S.M.; and Taman, A. R., Fuel, Vol. 90, PP. 42-47. (2011).

19-Gerpen, V.J.; Shanks, B.; and Pruszko, R.; (2004). National Renewable Energy Laboratory. PP. 1-100. 20- Almorfaq al alami.,Bioinformition.," Urtica dioica",(2011). 